\title{
Impact of deicing agents on heat network corrosion
}

\author{
Ivan Postnikov ${ }^{1, *}$, Valery Stennikov ${ }^{1}$, Vitaly Shamansky ${ }^{1}$, Alexander Kozlov ${ }^{1}$, Pavel Sokolov ${ }^{1}$ \\ ${ }^{1}$ Melentiev Energy Systems Institute of Siberian Branch of the Russian Academy of Sciences, Laboratory of Heat Supply Systems, \\ 664033 Irkutsk, Russia
}

\begin{abstract}
This research is caused by the need to make a scientific and technical analysis and obtain informed assessments of the consequences of deicing agents application to urban roads for the heat network infrastructure. The main objectives of this research were to assess the impact of the deicing agent on initiation and acceleration of corrosion of the heat network equipment (pipelines, nonmobile and sliding bearings), the effect of this corrosion on reliability of heat supply to consumers, and to determine the economic damage caused by the decline in service life of pipelines. In addition, the study identified additional circumstances of the detrimental effect of deicing agents on the heat network infrastructure associated with destructive changes in the concrete structures of the heat network thermal chambers.
\end{abstract}

\section{Introduction}

A significant part of urban heat networks (HN) in countries with cold climatic conditions are laid under roads, including long distance pipelines of large diameter. In this regard, every year the number of damaged pipelines due to the impact of deicing agents (DIA) increases.

Thus, informed assessments of the consequences of DIA application to urban roads for the $\mathrm{HN}$ infrastructure are needs. The main objectives of this research were to assess the impact of the DIA on initiation and acceleration of corrosion of the heat network equipment (pipelines, nonmobile and sliding bearings), the effect of this corrosion on reliability of heat supply to consumers, and to determine the economic damage caused by the decline in service life of pipelines.

To perform a physicochemical analysis, we selected fragments of salt deposits, pipelines and structures of thermal chambers affected by DIA related corrosion. The experiments were carried out using specialized and certified devices. All samples were obtained by inside examination of $\mathrm{HN}$ thermal chambers and sections of the district heating system (DHS) in Irkutsk. The techno economic analysis of the actual and predicted consequences induced DIA damage to the HN was conducted on the basis of statistical information from incident logs, data on the HN state and other documents, as well as reference data.

\section{Physicochemical study on the DIA related corrosion of $\mathrm{HN}$ equipment}

Sampling was carried out at the HN facilities of DHS of Irkutsk. Fig. 1 presents the photographs from some of the facilities exposed to DIA.
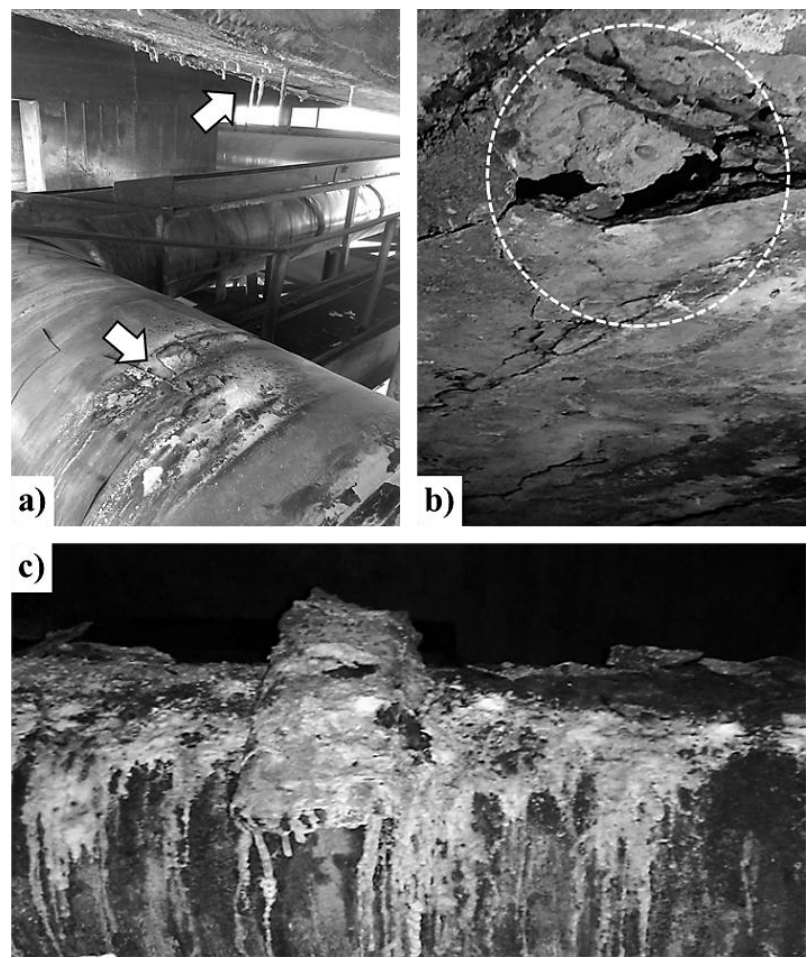

Fig. 1. View of the HN structures and pipelines exposed to DIA: a) concrete structures and pipeline under the Glazkovsky Bridge, Irkutsk (arrows point to mineral stalactites and the pipeline surface under them, that undergoes intensive destruction); b) concrete structure of one of the thermal chambers located under the road; c) the state of the thermalinsulation surface of the pipeline subjected to intensive impact of DIA.

In order to carry out a physicochemical analysis, we selected the fragments of salt deposits, thermal chamber structures (slabs, supporting beams), pipelines, fittings, 
thermal-insulation and anticorrosion layer of pipelines damaged by DIA related corrosion.
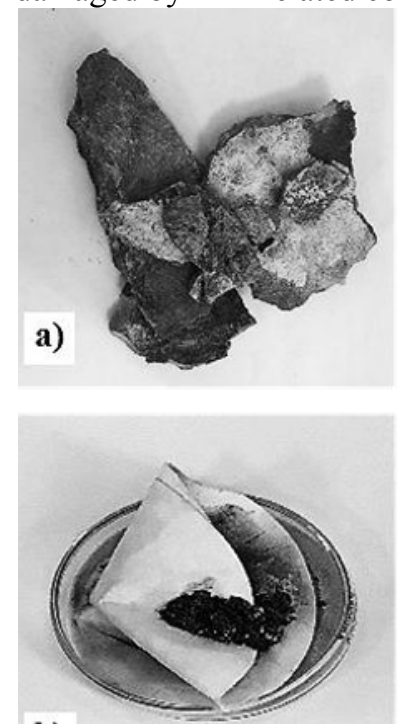

b)
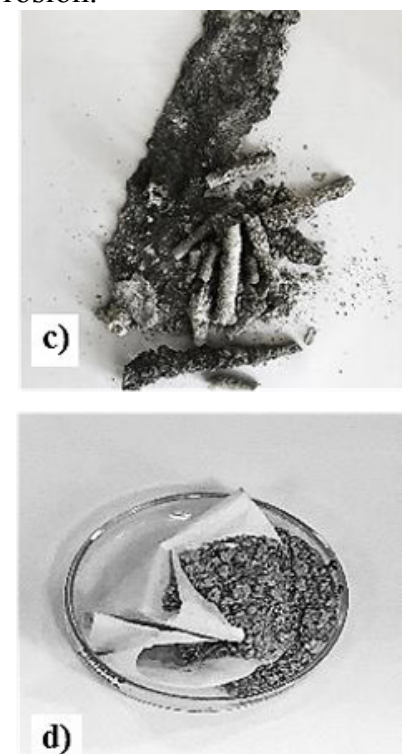

d)

Fig. 2. Samples for physicochemical analysis: a) and b) are the view of a sample of a concrete wall of thermal chamber of $\mathrm{HN}$ and undissolved residue obtained from its research; c) and d) are the view of a sample of the destroyed thermal insulation layer of $\mathrm{HN}$ and undissolved residue obtained from its investigation.

Fig. 2-a and 2-c demonstrate the samples prepared for laboratory studies (experiments), and Fig. 2-b and 2$\mathrm{d}-$ their undissolved residues after the experiment. The characteristics obtained as a result of the experiment with 4 different samples are presented in Table 1.

Table 1. Results of chemical analysis of solutions of the studied samples

\begin{tabular}{|c|c|c|c|c|c|}
\hline \multirow{2}{*}{\multicolumn{2}{|c|}{ Index }} & \multicolumn{4}{|c|}{ Sample No. } \\
\hline & & 1 & 2 & 3 & 4 \\
\hline \multicolumn{2}{|c|}{$\begin{array}{l}\text { Content of insoluble } \\
\text { part, \% }\end{array}$} & 1.0 & 87.3 & 0.1 & 84.7 \\
\hline \multicolumn{2}{|c|}{$\begin{array}{l}\text { Content of soluble } \\
\text { part, } \%\end{array}$} & 99.0 & 12.7 & 99.9 & 15.3 \\
\hline \multicolumn{2}{|c|}{$\begin{array}{l}\text { Concentration of } \\
\text { solution, } \%\end{array}$} & 4.2 & 0.62 & 4.8 & 0.78 \\
\hline \multicolumn{2}{|l|}{$\mathrm{pH}$} & 7.55 & 9.13 & 7.93 & 10.2 \\
\hline \multirow{6}{*}{ Composition } & $\mathrm{Ca}$ & 613 & 55 & 870 & 58 \\
\hline & $\mathrm{Mg}$ & 90 & 0.28 & 70 & 12 \\
\hline & $\mathrm{Na}$ & 17640 & 143 & 20417 & 4657 \\
\hline & $\mathrm{K}$ & 39 & 10 & 120 & 514 \\
\hline & $\mathrm{Cl}^{--}$ & 29419 & 215 & 33793 & 8392 \\
\hline & $\mathrm{SO}_{4}^{2--}$ & 357 & 185 & 3193 & 2013 \\
\hline
\end{tabular}

These characteristics are further used to theoretically analyze various stages of corrosion of the HN pipelines. One of the key factors that determines these processes is the $\mathrm{pH}$ index. The values of this index are presented in Table 1. They indicate an alkaline medium for all the samples under study.

Destruction of concrete structures. Chemically, concrete of the most common building brands is an alkaline composition with a $\mathrm{pH}$ value of $11-12$. When water percolates through concrete, lime is removed because of its solubility (up to $1.3 \mathrm{~g} / \mathrm{l}$ ) and, consequently, the strength of structure decreases, which subsequently leads to gradual destruction of the cement stone. Thus, the reduction in the amount of free lime by $20 \%$ leads to a considerable decrease in the mechanical properties of concrete structures [1-3]. At the same time, the branched fine capillary structure of concrete (capillary porosity of $7-10 \%$ ) makes the water filtration process rather slow.

The main components of DIA used on roads are sodium, calcium and magnesium chlorides. Magnesium chloride is affected by acid hydrolysis to a greater extent: $\mathrm{MgCl} 2+\mathrm{H} 2 \mathrm{O}=\mathrm{MgOHCl}+\mathrm{HCl}$. Getting to the surface of concrete structures, these components neutralize free lime in concrete and dissolve $\mathrm{CaCO} 3$. In this case, the expanded pores of concrete are filled with salt solutions, and after water evaporation, they are filled with solid crystals. The crystals are hygroscopic, therefore they absorb water and form crystalline hydrates, for example: $\mathrm{MgCl} 2.2 \mathrm{H} 2 \mathrm{O}, \mathrm{Na} 2 \mathrm{SO} 4.10 \mathrm{H} 2 \mathrm{O}$, etc. The pressure of crystallization and hydration arises, which leads to the formation of microcracks inside and on the surface of concrete. This phenomenon is greatly enhanced by cycles of climatic changes in temperature that lead to so-called concrete scaling, i.e. flaking or peeling of concrete surface. This process is clearly seen in Fig. 1-b.

Destruction of thermal insulation layer and anticorrosion coating. After penetration through concrete structures or surface due to washout of $\mathrm{Ca}(\mathrm{OH}) 2$ from the concrete, the DIA solution acquires alkaline properties. This is also confirmed by the results of a chemical analysis of samples taken from the pipelines and walls of thermal chambers (Table 1).

The thermal insulation of the considered pipelines is made on the basis of polyurethane foam, and the anticorrosion coating is based on the complex polyurethane coating. The heart of the solid polyurethane coating is the so-called prepolymer, which has two types of bonds responsible for the polymeric structure: the amide bond -CO-NH-R'- and the ester bond -CO-O-R-. The latter is unstable because the esters are highly susceptible to hydrolysis reaction, which leads to the breakdown of the bond and formation of an alcohol and an acid: R-CO-O-R'+ $\mathrm{H} 2 \mathrm{O}<=>\mathrm{R}-\mathrm{COOH}+\mathrm{R}^{\prime}-\mathrm{OH}$. It is known [4] that the rate of hydrolysis in alkaline medium compared to neutral medium $(\mathrm{pH}=7)$ is increased by $10^{(\mathrm{pH}(\mathrm{b})-7)}$ times, where $\mathrm{pH}(\mathrm{b})$ corresponds to the contacting solution. In the considered case the $\mathrm{pH}$ value of DIA measured after the salt is removed from the fragments of the destroyed thermal insulation, exceeds 10 units (see Table 1). This means that the rate of hydrolysis (i.e. the polymer destruction) rises at least by $10^{10-7}=1000$ times.

Electrochemical corrosion of metal pipelines under the effect of salt deposits. The main type of corrosion of metal pipelines is pitting corrosion, which is characterized by localized destructions observed in the cases where the corrosion rate in some areas is higher than in others [7-11]. It is also worth noting that in addition to pitting, pipelines are subject to stress corrosion cracking [12]. The effect of pitting processes can be seen on the fragments of the pipelines shown in 
Fig. 4. The picture "a" demonstrates pitting damage leading to through holes, while "b" one shows a section with remaining parts of polyurethane thermal insulation.

The rate of electrochemical corrosion [12-16] is proportional to the corrosion current, which, in accordance with Ohm's law, is proportional to the difference in potentials between the cathodic and anodic areas of the sur-face. In general, the surface to be corroded is a branched electrical (electrochemical) circuit in which the corrosion currents connect different anodic and cathodic areas. In this case, currents can flow between any differently charged micro-areas of a nonuniform metal surface. The existence of such heterogeneities is caused both by the nature of metal (heterogeneity of chemical composition) and by the mechanical state of metal (micro-inhomogeneity, dislocations, etc.).
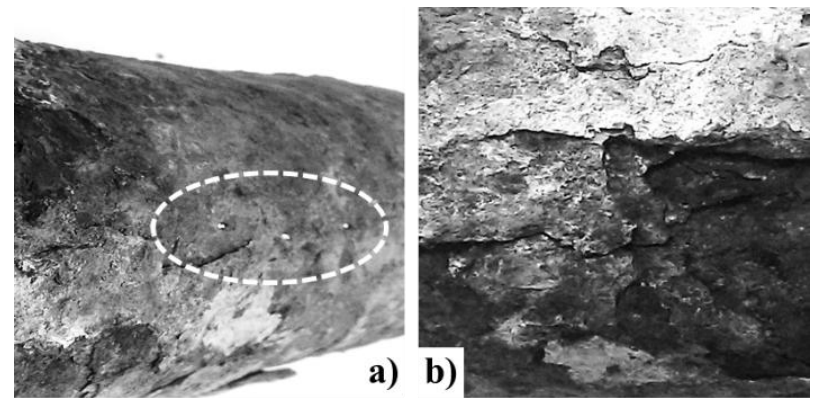

Fig. 3. Fragment of a pipe damaged by corrosion: a) section with pitting corrosion (dotted through holes are visible); b) section with surface corrosion and fragments of destroyed polyurethane thermal insulation.

In the anodic areas of the pipeline metal surface, in the absence of moisture, the iron ions remain on the surface and form the so-called electrical double layer with zero leakage current. However, in the presence of moisture, these ions are hydrated and removed from the structure of the double layer, which initiates their replenishment due to the formation of new ions, and the process of corrosion begins. This process consists of two successive reactions that result in well-known brown rust (ferric hydroxide): 1) $\mathrm{Fe} 2+2 \mathrm{OH}-=\mathrm{Fe}(\mathrm{OH}) 2$ and 2) $4 \mathrm{Fe}(\mathrm{OH}) 2+\mathrm{O} 2+2 \mathrm{H} 2 \mathrm{O}=4 \mathrm{Fe}(\mathrm{OH}) 3$. It is known that the presence of chlorides in the solution considerably accelerates corrosion $[8,9]$ because ferrous ions form highly soluble complexes with chloride ions $(\mathrm{FeCl} 2+$, $\mathrm{FeCl} 2+, \mathrm{FeCl} 3, \mathrm{FeCl} 4-)$. These complexes are highly strong and provoke dissolution of the iron hydroxide film that inhibits corrosion. As a result, corrosion of the pipeline surface is increased.

According to the assessment based on all the considered factors and conditions, the corrosion rate of $\mathrm{HN}$ pipelines exposed to DIA increases by approximately 7.5 times. As for the chemical corrosion under the effect of air oxygen [17], at this temperature this process is much slower than electrochemical corrosion [12].

\section{Techno economic and reliability analysis of DIA impact on HN pipeline}

The result of processing the data on the damage in $\mathrm{HN}$ of Irkutsk city associated with the use of DIA is shown in Table 2. The damage incidents that occurred in the nonheating season are mainly related to the hydraulic tests and have a long restoration period.

Table 2. DIA induced damage indices in HN of Irkutsk (2017)

\begin{tabular}{|l|c|}
\hline \multicolumn{1}{|c|}{ Index } & Value \\
\hline Total number of damage incidents in HN & 888 \\
\hline $\begin{array}{l}\text { Total number of damage incidents } \\
\text { associated with the use of DIA }\end{array}$ & $307(35 \%)$ \\
\hline $\begin{array}{l}\text { Total repair time of damage related to the } \\
\text { use of DIA, h }\end{array}$ & 3897 \\
\hline $\begin{array}{l}\text { Average repair time of damage associated } \\
\text { with the use of DIA, h }\end{array}$ & 13 \\
\hline $\begin{array}{l}\text { Total heat undersupply* due to DIA related } \\
\text { damage, Gcal }\end{array}$ & 12176 \\
\hline $\begin{array}{l}\text { Specific heat undersupply per 1 incident } \\
\text { associated with the use of DIA, Gcal }\end{array}$ & 40 \\
\hline $\begin{array}{l}\text { Total cost of repairing damaged areas, } \\
\text { thousand rub }\end{array}$ & 162043 \\
\hline $\begin{array}{l}\text { Total cost of repairing DIA related } \\
\text { damage*, thousand rub }\end{array}$ & $14578(9 \%)$ \\
\hline $\begin{array}{l}\text { Average cost of repairing damage due to the } \\
\text { use of DIA, thousand rub }\end{array}$ & 47 \\
\hline $\begin{array}{l}\text { Specific cost of repairing damage due to the } \\
\text { use of DIA, rub/Gcal }\end{array}$ & 1197 \\
\hline *indices calculated using the reference data [18] at
\end{tabular}

*indices calculated using the reference data [18] at a temperature difference of $130 / 70{ }^{\circ} \mathrm{C}$ and the specific friction pressure loss of $15 \mathrm{kgs} /\left(\mathrm{m}^{2} \mathrm{~m}\right)$

As can be seen from the Table 2, 307 damage incidents related to the use of DIA were recorded in $\mathrm{HN}$, which is $35 \%$ of the total number. It took 3897 hours to restore the areas damaged by DIA, with an average repair time of 13 hours. The total heat undersupply during the repair of damaged pipelines affected by DIA amounted to 12176 Gcal. Fig. 4 demonstrates the distribution of the pipeline repair duration and heat undersupply by month in 2017.

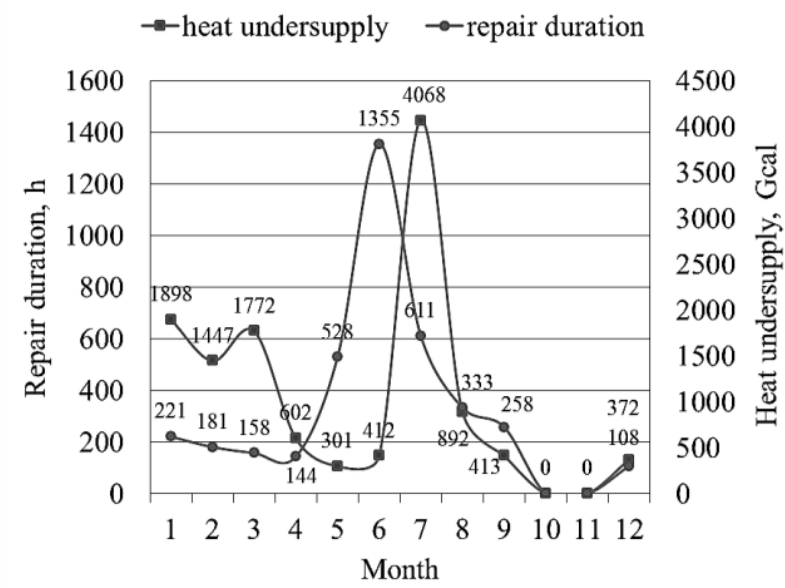

Fig. 4. Distribution of repair duration and heat undersupply due to $\mathrm{HN}$ pipeline damage caused by DIA in Irkutsk by month 2017

During this period, more complete reconstruction work is performed on damaged sections (branches) of HN. As seen from Fig. 4, this causes a considerable heat 
undersupply due to long-term interruptions of hot water supply (months 5-9).

Reliability analysis of HN considering DIA induced damage. Table 3 presents the results of an integrated reliability analysis based on the assessment of the availability factor (AF) for $\mathrm{HN}$ of Irkutsk in terms of pipeline damage associated with the use of DIA. In this case, AF is interpreted as an average value of the part of the heating period, during which the indoor air temperature in the customer's premises does not fall below the specified limit value $\left(t_{\min }\right)$ [19]. The assessment was carried out for three reliability criteria, corresponding to $t_{\min }=20^{\circ} \mathrm{C}, t_{\min }=18{ }^{\circ} \mathrm{C}$ and $t_{\min }=12$ ${ }^{\circ} \mathrm{C}$. It took into account the slack time related to heat accumulation in buildings and employed nomographic charts of cooling of residential premises [20].

Table 3. Integrated reliability analysis of HN in Irkutsk, considering pipeline damage related to the use of DIA (2017)

\begin{tabular}{|l|c|c|c|}
\hline \multirow{2}{*}{\multicolumn{1}{|c|}{ Index }} & \multicolumn{3}{|c|}{ Criteria $t_{\min }$} \\
\cline { 2 - 4 } & $20^{\circ} \mathrm{C}$ & $18^{\circ} \mathrm{C}$ & $12^{\circ} \mathrm{C}$ \\
\hline Total number of disconnections & 127 & 32 & 7 \\
\hline Total time of disconnections, $\mathrm{h}$ & 811 & 305 & 89 \\
\hline Average time of repair, h & 6.4 & 6.4 & 6.4 \\
\hline Availability factor & 0.86 & 0.95 & 0.98 \\
\hline $\begin{array}{l}\text { Required reduction in } \\
\text { disconnection time, } \mathrm{h}\end{array}$ & 637 & 131 & 0 \\
\hline $\begin{array}{l}\text { Required reduction in the } \\
\text { number of damage incidents }\end{array}$ & 100 & 21 & 0 \\
\hline
\end{tabular}

Note: all the indicators are calculated for a heating period of $5784 \mathrm{~h}$; disconnection is interpreted as an interruption of heat supply to consumers in compliance with the accepted criterion; $\mathrm{AF}$ is calculated for DHS only with respect to DIA related damage; minimum values for ensuring standard reliability that corresponds to the value of AF 0.97

According to [21], the standard value of AF is 0.97 . As seen from Table 3, the required level of reliability under the existing number of DIA induced damage incidents is achieved only if the minimum indoor air temperature of the consumer is at a level of $12{ }^{\circ} \mathrm{C}$. Based on the calculations, the necessary conditions were also established to ensure AF standard value for the specified criteria (Table 3).

Analysis of DIA induced corrosion effect on service life of HN pipelines and their replacement cost overruns. A physicochemical analysis of the increase in DIA induced corrosion intensity in $\mathrm{HN}$ (section 2) allows us to confirm the acceleration of the pipeline wear by at least 7.5 times. Taking into account this factor, the data on the length of the Irkutsk HN located under the roads, and the "age" structure of the networks, we made a forecast (up to 2024) of HN pipelines wear and tear (Fig. 5). The starting point of the forecast (2017) is $62 \mathrm{~km}$ (14\% of the total length of $\mathrm{HN}$ in the city) and corresponds to the length of worn-out $\mathrm{HN}$ under the roads.

According to the obtained results, without annual replacement of pipelines, the length of worn-out networks located under the roads by 2024 will be $73 \mathrm{~km}$ ( $16 \%$ of the total HN length) disregarding DIA impact, and $87 \mathrm{~km}(19 \%)$, considering the effect of DIA. At the rate of network replacement equal to that in 2017 (3.9 $\mathrm{km} /$ year) and the absence of DIA, the deterioration of the networks will decrease to $46 \mathrm{~km}(10 \%)$ by 2024 , and in the case of DIA use, its level will practically not change (Fig. 5). For the level of wear achieved at the current replacement rate and the impact of DIA, it is necessary to increase the rate of pipeline replacement to $5.8 \mathrm{~km}$.

Based on the indices of the pipeline restoration rates and data on the cost of the pipelines replacement, we compared the expected cost of replacing the worn $\mathrm{HN}$ in Irkutsk until 2024 with and without consideration of DIA effect. The level of wear at the end of the considered period was taken equal to $46 \mathrm{~km}(10 \%)$, which corresponds to the current replacement rate of $3.9 \mathrm{~km}$ without DIA effect. The results of this assessment are given in Table 4.

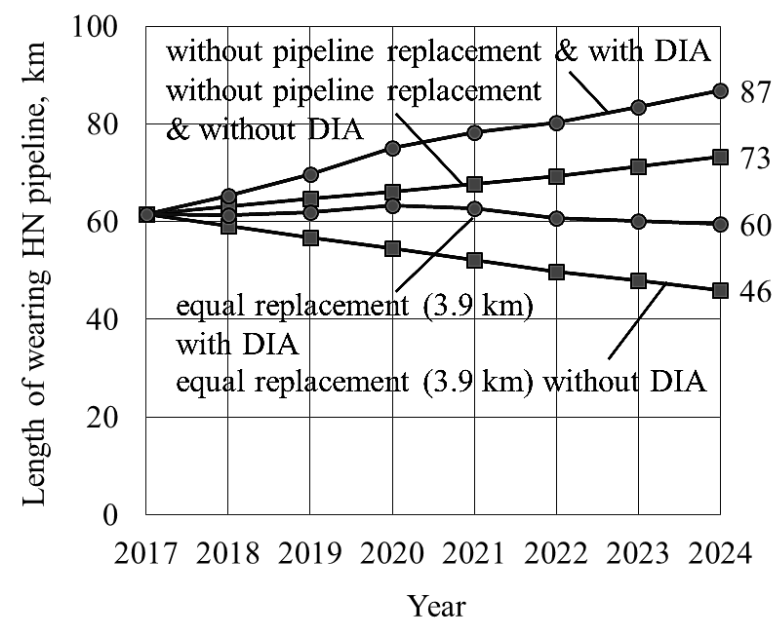

Fig. 5. The forecast dynamics of wear and tear in Irkutsk $\mathrm{HN}$ at various annual rates of pipeline replacement with and without using of DIA

The existing level of the pipeline replacement costs disregarding DIA is about 347 million rub/year. At the same time, maintaining the replacement rate of 5.8 $\mathrm{km} /$ year that provides the same level of wear at the end of the calculation period $(46 \mathrm{~km})$ will require much higher costs that are estimated at about 516 million rub/year. Thus, the cost overruns related to the replacement of the under road pipelines worn-out due to DIA induced corrosion, are quite significant and amount to $49 \%$, which corresponds to 169 million rub/year.

Table 4. Comparative (with and without the consideration of DIA) forecast of the replacement cost for Irkutsk worn-out HN located under roads, with their length reduction to $46 \mathrm{~km}$ $(10 \%)$ by 2024 , with the cumulative total, million rub

\begin{tabular}{|l|c|c|c|c|c|c|c|}
\hline \multirow{2}{*}{ Index } & \multicolumn{7}{|c|}{ Year } \\
\cline { 2 - 9 } & 2018 & 2019 & 2020 & 2021 & 2022 & 2023 & 2024 \\
\hline $\begin{array}{l}\text { Replacement } \\
\text { rate of 3.9 km }\end{array}$ & 347 & 693 & 1040 & 1387 & 1734 & 2080 & 2427 \\
\hline $\begin{array}{l}\text { Replacement } \\
\text { rate of 5.8 km }\end{array}$ & 516 & 1031 & 1547 & 2062 & 2578 & 3094 & 3609 \\
\hline Overrun & 169 & 338 & 507 & 676 & 845 & 1013 & 1182 \\
\hline
\end{tabular}

The cost in Table 4 for each subsequent year is shown in the format of a cumulative total, i.e. includes the sum of costs for the previous years. As a result, the 
value of the cost for 2024 corresponds to the amount of costs for the entire period in question.

\section{Conclusions}

The main objectives of research were to comprehensively assess the effect of DIA on corrosion of $\mathrm{HN}$, including a physicochemical analysis of corrosion processes and a techno economic analysis of the consequences of corrosion related damage to $\mathrm{HN}$ pipelines. The studies were carried out at $\mathrm{HN}$ of Irkutsk city, and the samples taken directly from thermal chambers of $\mathrm{HN}$ were used as initial materials for a laboratory analysis.

The physicochemical analysis of the samples revealed the indisputable factors of the destructive effect of DIA on polyurethane anticorrosion and thermal insulation coatings of the pipelines. The calculations demonstrated at least a 1000 -fold increase in the rate of hydrolysis, i.e. destruction of polymer insulation, under the operating temperatures of $\mathrm{HN}$ pipelines $\left(70-130^{\circ} \mathrm{C}\right)$. Moreover, the process of corrosion-related wear of metal pipelines is accelerated by about 7.5 times.

The techno economic analysis of the consequences of DIA use for HN pipelines involved generalized and structured statistical data on the incidents in $\mathrm{HN}$ of the city. Based on the calculations using statistical analysis and interpolation methods, we obtained the indices necessary to assess the consequences of pipeline damage caused by DIA in terms of the duration of repairs, heat undersupply, restoration costs, etc. The findings indicate that with the existing number of such damage incidents, the required level of reliability is achieved only for the minimum indoor air temperature at a level of $12{ }^{\circ} \mathrm{C}$. Additional reliability measures will be necessary to meet higher comfort requirements of the consumer in case of pipeline accidents.

The decrease in the service life of the Irkutsk under road HN pipelines damaged by DIA related corrosion has been assessed. The assessment indicates that with the current rates of pipelines replacement, the level of their wear and tear related to DIA application is not reduced. Moreover, the reduction in $\mathrm{HN}$ depreciation associated with DIA effect will require a significant increase in funding their reconstruction.

In conclusion, it is worth noting that the conducted studies confirmed the negative effect of DIA on HN of the city. The scale and extent of this effect call for additional in depth investigation of this issue and measures to eliminate the negative corrosive effect of DIA on the heat supply facilities of the city.

The study was carried out at Melentiev Energy Systems Institute SB RAS within the framework of research projects III.17.5.3 №AAAA-A17-117030310450-3 and III.17.1.2 №AAAA-A17-117030310448-0 of the Program for Fundamental Research of SB RAS, and involved the equipment of the Multiple-access Center "High temperature plant" at Melentiev Energy Systems Institute SB RAS.

\section{References}

1. Bazhenov Yu.M. Technology of concrete / M.: Publishing house of the ASV, 2002, $500 \mathrm{p}$.

2. Ramachandran V., Feldman R., Beaudoin J. Concrete Science. Physicochemical concrete study / M.: Stroiizdat, 1986, 279 p.

3. Gorchakov GI, Bazhenov Yu.M. Building Materials / Moscow: Stroiizdat, 1986, 689 p.

4. Lurie Yu.Yu. Handbook of Analytical Chemistry / M $\therefore$ Khimiya, 1971, $455 \mathrm{p}$.

7. Ulig G. G., Revi R.U. Corrosion and fight against it / Leningrad: Khimiya, 1989, $456 \mathrm{p}$.

8. Corrosion, Volume 1: Metal / Environment Reactions / Ed. L.L. Shreir, Elsevier Ltd., 1976, 1232 p.

9. Kruger J., Begum S. Corrosion of Metals: Overview // Reference Module in Materials Science and Materials Engineering, 2016.

10. Chaves Igor A., Melchers Robert E. Pitting corrosion in pipeline steel weld zones // Corrosion Science, 2011, vol. 53 (12), p. 4026-4032.

11. Hunkler F., Frankel G.S., Bohni H. On the mechanism of localized corrosion // Corrosion, 1987, vol. 43, p. 189-191.

12. Vorobieva G.Ya. Corrosion Resistance of Materials in Aggressive Media of Chemical Production / M.: Khimiya, 1975, 816 p.

13. Zhuk N.P. A course in the theory of corrosion and protection of metals / M : Metallurgy, 1976, $472 \mathrm{p}$.

14. Maocheng Y.A.N., Jin X.U., Libao Y.U., Tangqing W.U., Cheng S.U.N., Wei K.E. EIS analysis on stress corrosion initiation of pipeline steel under disbonded coating in near-neutral $\mathrm{pH}$ simulated soil electrolyte // Corrosion Science, 2016, vol. 110, p. 23-34.

15. Guo H.X.. Lu B.T., Luo J.L. Interaction of mechanical and electrochemical factors in erosioncorrosion of carbon steel // Electrochimica Acta, 2005, vol. 51 (2), p. 315-323.

16. Lu B.T., Luo J.L. Synergism of Electrochemical and Mechanical Factors in Erosion-Corrosion // J. Phys. Chem. B, 2006, vol. 110(9), p. 4217-4231.

17. Kusmierek E., Chrzescijanska E. Atmospheric corrosion of metals in industrial city environment // Data in Brief, 2015, vol. 3, p. 149-154.

18. Design of heat networks. Reference book of designer. Ed. by Nikolaev A.A. / M.: Publishing House for Literature on Construction, 1965, $400 \mathrm{p}$.

19. Reliability of heating systems / Reference book. Ed. by Sennova E.V. Novosibirsk: Science, 2000, 360 p.

20. Areshkin A.A., Moskalenko A.V., Gorobets N.V. General principles of heat supply backup in heating systems // Novosti Teplosnabzhenia (Heat Supply News), 2011, vol. 1, p. 26-34.

21. Construction Norms and Regulations P 41-02-2003 "Heat networks" / M: Ministry of Regional Development of the Russian Federation, 2012, 75 p. 